\title{
HLA class II DRB1 and DQB1 allelic polymorphism and sclerosing lymphocytic lobulitis of the breast
}

Andrew H S Lee, Adrian C Bateman, Stephen J Turner, Jeffrey M Theaker, W Martin Howell

\begin{abstract}
Background-Sclerosing lymphocytic lobulitis is an inflammatory disorder of the breast. The pattern of inflammation, expression of HLA class II DR by breast epithelium and association with autoimmune disorders, particularly insulin dependent diabetes mellitus (IDDM), together suggest an autoimmune aetiology.
\end{abstract}

Aim-To test the hypothesis that susceptibility to sclerosing lymphocytic lobulitis may be linked to patient HLA class II DRB1 alleles, particularly DRB1*03 and DRB $1{ }^{\star} 04$, suggested by a previous small uncontrolled study.

Methods-HLA class II DRB1 and DQB1 genotypes were compared between a series of 28 sclerosing patients with 1 ymphocytic lobulitis and 300 controls, using polymerase chain reaction (PCR) based typing of DNA extracted from formalin fixed, paraffin embedded biopsies.

Results-Results from the 28 patients (nine with IDDM) showed an increased frequency of $D R B 1{ }^{\star} 04$ compared with controls $(61 \% v 33 \%, p=0.01)$, but no significant differences for other genotypes. In patients with IDDM, the frequencies of DRB ${ }^{\star}{ }^{\star 04} \quad(89 \%, \quad p=0.001) \quad$ and DQB1*0302 $(100 \%$ v 21\%, p = 0.0001)-a genotype that is in linkage disequilibrium with DRB $1{ }^{\star} 04$-were increased compared with controls. However, in the patients without IDDM, the frequencies of DRB1*04 (50\%) and DQB1*0302 (14\%) were not significantly increased.

Conclusions-The increased frequency of HLA DRB1*04 in sclerosing lymphocytic lobulitis appears to reflect its association with IDDM, a condition in which DRB1*04 is increased in frequency. (f Clin Pathol 1999;52:445-449)

Keywords: sclerosing lymphocytic lobulitis; breast; HLA antigens; histocompatibility testing

Sclerosing lymphocytic lobulitis is a recently recognised inflammatory disorder of the breast. It is characterised by clusters of $\mathrm{B}$ and $\mathrm{T}$ lymphocytes in and around lobules and ducts, and around blood vessels, with increased expression of class II human leucocyte antigens (HLA) by the lobular and ductal epithelium, fibrosis, often with epithelioid fibroblasts, and lobular atrophy. ${ }^{12}$ The pattern of inflammation resembles that seen in Hashimoto thyroiditis, ${ }^{3}$ myoepithelial sialadenitis, ${ }^{4}$ and insulin dependent diabetes mellitus. ${ }^{5}$ Sclerosing lymphocytic lobulitis is associated with autoimmune diseases, particularly with insulin dependent diabetes mellitus and thyroiditis. ${ }^{1267}$ The pattern of inflammation, expression of HLA class II by breast epithelium, and association with autoimmune diseases together suggest an autoimmune aetiology.

Many $\mathrm{T}$ lymphocyte mediated diseases, including autoimmune conditions such as ankylosing spondylitis, rheumatoid arthritis, and diabetes mellitus, have been shown to occur more frequently among individuals possessing certain HLA alleles. ${ }^{8}$ HLA class I molecules are expressed on the surface of almost all nucleated cells and generally present endogenous antigens to CD8+ T lymphocytes. HLA class II molecules are expressed on a more restricted range of cells including those of macrophage/monocyte lineage and usually present exogenous antigens to $\mathrm{CD} 4+\mathrm{T}$ lymphocytes. ${ }^{9}$ HLA molecules are encoded within the major histocompatibility complex by the most polymorphic loci within the human genome; for example, there are 197 DRB1 and 35 DQB1 alleles currently identified. ${ }^{10}$ Allelic polymorphisms within the HLA encoding genes change the conformation of the antigen binding groove and therefore alter the efficiency of antigen binding and presentation by the HLA molecules. ${ }^{11}$ This may result in HLA associated interindividual differences in specific $T$ cell responsiveness to particular antigens. ${ }^{12} 13$

Two uncontrolled studies have performed HLA typing on patients with sclerosing lymphocytic lobulitis using serological methods. In one study of seven patients (two with type 1 diabetes mellitus), selected by their biopsy appearances, three patients were of HLA-DR3 phenotype, and two were HLA-DR4. ${ }^{1}$ In a second study of 10 patients, who all also had insulin dependent diabetes mellitus, four patients were found to be HLA-DR3, and six HLA-DR4. ${ }^{6}$

The aim of this study was to test the hypothesis that patients with sclerosing lymphocytic lobulitis have an increased frequency of HLADR3 and DR4, through the comparison of HLA class II DRB1 and DQB1 genotype frequencies in a larger series of patients with those of healthy controls, using polymerase chain reaction (PCR) based typing of DNA extracted from formalin fixed, paraffin embedded biopsies. Compared with serological typ- 
Table 1 Comparison of serological type and genotype for selected HLA alleles

\begin{tabular}{ll}
\hline Serological type & Genotype \\
\hline $\mathrm{DR} 3$ & $\mathrm{DRB} 1^{\star} 03$ \\
$\mathrm{DR} 4$ & $\mathrm{DRB} 1^{\star} 04$ \\
$\mathrm{DQ} 2$ & $\mathrm{DQB} 1^{\star} 02$ \\
$\mathrm{DQ} 8$ & $\mathrm{DQB} 1^{\star} 0302$ \\
\hline
\end{tabular}

Note that the first three genotypes can be subdivided into additional alleles by high resolution DNA typing.

ing, this method is more accurate, of higher resolution, ${ }^{14}$ and can be used on archival tissue..$^{1516}$ We were also interested in the frequency of two genotypes associated with insulin dependent diabetes mellitus, HLADQB $1{ }^{\star} 02$ and $\mathrm{DQB} 1{ }^{\star} 0302$, because of the association of sclerosing lymphocytic lobulitis with diabetes, and the known linkage disequilibrium between HLA- DQB $1^{\star} 02$ and $\mathrm{DRB} 1{ }^{\star} 03$, and between $\mathrm{DQB} 1{ }^{\star} 0302$ and $\mathrm{DRB} 1{ }^{\star} 04 .{ }^{17}$ To aid comparison between previous studies that used serological HLA typing and the present study, the corresponding nomenclature for serological typing and genotyping for the alleles of interest is shown in table 1 .

\section{Methods}

SELECTION OF PATIENTS

Biopsies with a diagnosis of sclerosing lymphocytic lobulitis from the Hedley Atkins Pathology Laboratory, ICRF Clinical Oncology Unit, Guy's Hospital (1985 to 1996) and the Department of Histopathology, Southampton University Hospitals (1990 to 1998) were reviewed; eight had been included in previous studies of this disorder. ${ }^{18}$ To be included in this study, the biopsy had to show the characteristic pattern of inflammation: circumscribed clusters of lymphocytes centred on lobules and vessels. In many cases immunohistochemistry was performed, confirming that these were B and T lymphocytes. Lobular atrophy, fibrosis, and epithelioid fibroblasts were often present.

All 28 patients with sclerosing lymphocytic lobulitis fulfilling the above criteria had HLA class II DRB and DQB1 genotyping performed. Control HLA class II genotype frequencies were obtained from the routine typing of cadaveric and non-cadaveric solid organ and bone marrow transplant donors, with 300 control individuals typed for the HLA DRB1 and DQB1 loci, using the same HLA class II genotyping method.

The records of the patients were reviewed to identify the mode of presentation and any associated disorders, particularly insulin dependent diabetes mellitus and thyroid disease.

HLA CLASS II GENOTYPING

DNA extraction

Two to five $20 \mu \mathrm{m}$ paraffin sections were cut from each case under investigation. The separated wax embedded chips were dewaxed in xylene (Merck) and xylene-ethanol washes. The resulting cellular material was pelleted, washed in absolute ethanol (James Burrough $(\mathrm{FAD}))$, and dried at $50^{\circ} \mathrm{C}$. The dried pellet was then incubated at $55^{\circ} \mathrm{C}$ overnight in $100 \mu \mathrm{l}$ lysis mix (100 mM Tris-HCl, pH 8 (Sigma), 4 mM EDTA (Merck), 0.45\% NP40 (Sigma), and $0.45 \%$ Tween 20 (Sigma)) containing 6 $\mathrm{mg} / \mathrm{ml}$ proteinase $\mathrm{K}$ (Sigma). The mixture was then boiled for five minutes to inactivate the proteinase $\mathrm{K}$.

PCR amplification and sequence specific oligonucleotide probe (PCR-SSOP) genotyping This technique was used to provide HLA DRB1 , 3, 4, and 5 and DQB1 genotypes in 20 of the 28 cases in this study. This was achieved by PCR amplification of the second (variable) exon of the class II genes in question, using PCR primers and amplification conditions as described for the 11 th International Histocompatibility Workshop, ${ }^{19}$ and as routinely used for archival surgical biopsy genotyping and previous HLA-disease association studies in our laboratory. ${ }^{15}{ }^{16}$ This PCR-SSOP typing system provides medium resolution HLA-DRB and DQB1 typing. Two microlitres of each DNA preparation were used per PCR. Following dot blot preparation of amplified DNAs, SSOP typing was performed using panels of oligonucleotide probes selected from the 11th Workshop panel; 18 DRB probes and 18 DQB probes were used. All probes were 5 ' end labelled with digoxigenin (DIG-11-ddUTP (Boehringer Mannheim)). Dot blot membrane blocking, prehybridisation, hybridisation, and stringency washing were performed according to the protocol of Nevinny-Stickel et al. ${ }^{20}$ Detection of bound probe was achieved using the chemiluminescent substrate CSPD (Boehringer Mannheim), according to the manufacturer's protocol, and visualised following 10 to 40 minute exposure to $x$ ray film.

Nested PCR and sequence specific primer (PCR-SSP) HLA DRB genotyping

This technique was used to provide HLA DRB1,3, 4, and 5 genotypes in eight of the 28 cases in this study, in which insufficient intact DNA was extractable from the formalin fixed, paraffin embedded tissues for successful PCRSSOP genotyping. PCR amplification of the second (variable) exon of the HLA-DRB1, 3, 4 , and 5 genes was performed using PCR primers and amplification conditions as described for the 11th International Histocompatibility Workshop, ${ }^{19}$ with minor modifications. A second phase of PCR using 23 sequence specific primer pairs for HLA-DRB1, 3,4 , and 5 genes was then performed. The details of the PCR mixes and conditions for both amplification steps have been described previously. ${ }^{21}$ PCR products were visualised by running the entire reaction mix on a $2 \%$ agarose gel.

STATISTICAL ANALYSIS

The frequencies of each HLA DRB1 and DQB1 genotypes were calculated for the patients with sclerosing lymphocytic lobulitis and were compared with those obtained in the control individuals typed for each locus. The null hypothesis was tested for each comparison using $\chi^{2}$ analysis with Yate's correction. Two tailed probability values were calculated with 
Table 2 Comparison of genotype frequencies in sclerosing lymphocytic lobulitis patients and controls

\begin{tabular}{|c|c|c|c|}
\hline \multirow[b]{2}{*}{ Genotypes } & \multicolumn{2}{|c|}{ Frequencies of genotypes (\%) } & \multirow[b]{2}{*}{$\chi^{2}$ Analysis } \\
\hline & $\begin{array}{l}\text { Sclerosing lymphocytic } \\
\text { lobulitis }\end{array}$ & Controls & \\
\hline DRB1 & $\mathrm{n}=28$ & $\mathrm{n}=300$ & \\
\hline $\mathrm{DRB} 1{ }^{\star} 01$ & $3(11 \%)$ & $60(20 \%)$ & $\chi^{2}=0.89, p=0.50$ \\
\hline $\mathrm{DRB} 1{ }^{\star} 03$ & $8(29 \%)$ & $87(29 \%)$ & \\
\hline $\mathrm{DRB} 1{ }^{\star} 04$ & $17(61 \%)$ & $98(33 \%)$ & $\chi^{2}=7.66, \mathrm{p}=0.006, \mathrm{p}_{\mathrm{c}}=0.01$ \\
\hline $\mathrm{DRB} 1{ }^{\star} 07$ & $2(7 \%)$ & $70(30 \%)$ & $\chi^{2}=3.03, p=0.06, p_{c}=0.78$ \\
\hline $\mathrm{DRB} 1{ }^{\star} 08$ & $3(11 \%)$ & $24(8 \%)$ & \\
\hline $\mathrm{DRB} 1{ }^{\star} 09$ & $1(4 \%)$ & $6(2 \%)$ & \\
\hline $\mathrm{DRB} 1{ }^{\star} 10$ & $0(0 \%)$ & $6(2 \%)$ & \\
\hline $\mathrm{DRB} 1 \star 11$ & $4(14 \%)$ & $47(16 \%)$ & \\
\hline $\mathrm{DRB} 1{ }^{\star} 12$ & $0(0 \%)$ & $8(3 \%)$ & \\
\hline $\mathrm{DRB} 1 * 13$ & $3(11 \%)$ & $71(24 \%)$ & $\chi^{2}=1.77, p=0.18$ \\
\hline $\mathrm{DRB} 1 \star 14$ & $2(7 \%)$ & $17(6 \%)$ & \\
\hline $\mathrm{DRB} 1{ }^{\star} 15$ & $7(25 \%)$ & $67(22 \%)$ & \\
\hline $\mathrm{DRB} 1 \star 16$ & $0(0 \%)$ & $4(1 \%)$ & \\
\hline$D Q B 1$ & $\mathrm{n}=20$ & $\mathrm{n}=300$ & \\
\hline $\mathrm{DQB} 1{ }^{\star} 02$ & $7(35 \%)$ & $129(43 \%)$ & \\
\hline DQB1*0301 & $7(35 \%)$ & $100(33 \%)$ & \\
\hline $\mathrm{DQB} 1{ }^{\star} 0302$ & $8(40 \%)$ & $64(21 \%)$ & $\chi^{2}=2.75, p=0.08, p_{c}=0.16$ \\
\hline $\mathrm{DQB} 1{ }^{\star} 0303$ & $1(5 \%)$ & $25(8 \%)$ & \\
\hline DQB1*04 & $1(5 \%)$ & $21(7 \%)$ & \\
\hline $\mathrm{DQB} 1{ }^{\star} 05$ & $4(20 \%)$ & $83(28 \%)$ & \\
\hline DQB $1 * 06$ & $6(30 \%)$ & $119(40 \%)$ & \\
\hline
\end{tabular}

$\left(p_{c}\right)$ and without $(p)$ corrections for the number of comparisons made at each HLA class II locus. For HLA-DRB ${ }^{\star} 03$ and 04 , and DQB $1^{\star} 02$ and 0302 a correction factor of 2 was used as these alleles were selected to be of interest before analysis. For all other genotypes a more conservative correction was applied, according to the number of comparisons (alleles) made at each locus: 13 for the HLA DRB1 loci and seven for the HLA DQB1 loci. ${ }^{22}$ Relative risks were calculated where a particular genotype or allele showed a significantly increased incidence (after correction for the number of comparisons made) within the study group or subgroup, compared with controls. $^{22}$

The frequencies of selected genotypes were compared in patients with and without insulin dependent diabetes mellitus. The frequencies of these genotypes in these two subgroups of patients with sclerosing lymphocytic lobulitis were separately compared with controls. As the numbers were smaller in this subgroup analysis, Fisher's exact test was used.

\section{Results}

The 28 patients with sclerosing lymphocytic lobulitis were all female, and from 25 to 66 years old, with 19 patients in their thirties. Nine patients had insulin dependent diabetes mellitus of from nine to 29 years' duration (in one patient the duration was not available). One patient had vitiligo, one had a family history of insulin dependent diabetes mellitus, and one diabetic patient also had Hashimoto thyroiditis. All presented with a breast mass or nodularity. The clinical diagnosis was of a benign lesion in 23 patients. The clinical differential diagnosis included carcinoma in four patients and sclerosing lymphocytic lobulitis in two. The pathological changes in patients with and without diabetes mellitus were similar; epithelioid fibroblasts were seen in both groups.

The frequencies of the different HLA-DRB1 and DQB1 genotypes in the patients with sclerosing lymphocytic lobulitis and the controls are shown in table 2 . In patients with sclerosing lymphocytic lobulitis there were increased frequencies compared with controls of DRB $1^{\star} 04$ (relative risk 1.86, 95\% confidence interval 1.32 to 2.61 ); and of DQB $1^{\star} 0302$, although the latter increase was not statistically significant after correction. The frequencies of HLA$\mathrm{DRB} 1{ }^{\star} 03$ and HLA-DQB $1{ }^{\star} 02$ were not increased in patients with sclerosing lymphocytic lobulitis. Four patients were HLA typed by serology as part of a previous study. ${ }^{1}$ There was complete agreement between serological typing and genotype for the 13 alleles for which both typing data were available (one DQ allele could not be characterised by serology in one patient and DQB1 genotyping could not be performed in another patient).

The two genotypes that were increased, $\mathrm{DRB} 1{ }^{\star} 04$ and $\mathrm{DQB} 1{ }^{\star} 0302$, are in linkage disequilibrium with each other ${ }^{17}$ and are associated with insulin dependent diabetes mellitus. ${ }^{23}$ We therefore compared the frequencies of selected genotypes in patients with sclerosing lymphocytic lobulitis who did and did not have diabetes (table 3 ). In patients with sclerosing lymphocytic lobulitis, both $\mathrm{DRB} 1{ }^{\star} 04$ and DQB $1{ }^{\star} 0302$ were seen at higher frequency in those with diabetes. In patients with diabetes, the frequencies of $\mathrm{DRB} 1^{\star} 04 \quad(89 \%)$ and DQB1*0302 (100\%) were significantly increased compared with controls. However, in the patients without diabetes, the frequencies of DRB $1^{\star} 04(50 \%)$ and DQB $1{ }^{\star} 0302(14 \%)$ were not significantly increased.

\section{Discussion}

We found a strong association between sclerosing lymphocytic lobulitis and long standing insulin dependent diabetes mellitus, in agreement with previous series. ${ }^{1267}$ Other autoimmune diseases occurred much less frequently. The pathological features in patients with and without diabetes mellitus were similar. It has been suggested that epithelioid fibroblasts are specific for "diabetic mastopathy," ${ }^{24}$ but we

Table 3 Comparisons of frequencies of selected genotypes in sclerosing lymphocytic lobulitis patients with and without insulin dependent diabetes mellitus, and with control patients

\begin{tabular}{|c|c|c|c|c|c|c|}
\hline \multirow[b]{2}{*}{ Genotype } & \multicolumn{2}{|c|}{ Insulin dependent diabetes mellitus } & \multirow{2}{*}{$\begin{array}{l}\text { Diabetic v } \\
\text { non-diabetic }\end{array}$} & \multirow[b]{2}{*}{ Controls } & \multirow{2}{*}{$\begin{array}{l}\text { Diabetic v } \\
\text { control }\end{array}$} & \multirow{2}{*}{$\begin{array}{l}\text { Non-diabetic v } \\
\text { control }\end{array}$} \\
\hline & Present & Absent & & & & \\
\hline $\mathrm{DRB} 11^{\star} 03$ & $4 / 9(44 \%)$ & $4 / 19(21 \%)$ & $\mathrm{p}=0.37$ & $29 \%$ & $\mathrm{p}=0.46$ & $p=0.60$ \\
\hline $\mathrm{DRB} 1{ }^{\star} 04$ & $8 / 9(89 \%)$ & $9 / 18(50 \%)$ & $\mathrm{p}=0.09$ & $33 \%$ & $\mathrm{p}=0.001$ & $\mathrm{p}=0.20$ \\
\hline $\mathrm{DQB} 1{ }^{\star} 02$ & $2 / 6(33 \%)$ & $5 / 14(36 \%)$ & $\mathrm{p}=1.0$ & $43 \%$ & $\mathrm{p}=0.70$ & $\mathrm{p}=1.0$ \\
\hline DQB $1{ }^{\star} 0301$ & $2 / 6(33 \%)$ & $5 / 14(36 \%)$ & $\mathrm{p}=1.0$ & $33 \%$ & $\mathrm{p}=1.0$ & $\mathrm{p}=1.0$ \\
\hline DQB $1{ }^{\star} 0302$ & $6 / 6(100 \%)$ & $2 / 14(14 \%)$ & $\mathrm{p}=0.0007$ & $21 \%$ & $\mathrm{p}=0.0001$ & $\mathrm{p}=0.74$ \\
\hline
\end{tabular}

Fisher's exact test used for all comparisons. 
found epithelioid fibroblasts in patients both with and without diabetes mellitus, in agreement with a previous study. ${ }^{25}$ The similarity of the pathology in both groups suggests that they are the same disorder and that a general pathological term such as sclerosing lymphocytic lobulitis or lymphocytic mastopathy is preferable to diabetic mastopathy.

Patients with sclerosing lymphocytic lobulitis who have had a series of biopsies often show a progression from dense inflammation to increasing lobular atrophy and fibrosis and decreasing inflammation. ${ }^{1}$ The changes seen later in the disease (lobular atrophy and fibrosis with little inflammation) are not specific to this disorder. The most characteristic pathological feature is the circumscribed perilobular and perivascular lymphocytic aggregates. We therefore only included cases with this characteristic pattern of inflammation.

Two previous small, uncontrolled studies of patients with sclerosing lymphocytic lobulitis using serological phenotyping suggested that the disorder may be associated with HLA-DR3 and DR4. ${ }^{16}$ We tested this hypothesis using a larger series of patients with a more precise genotyping method and a large control group, finding an association with HLA-DRB $1^{\star} 04$ but not with $\mathrm{DRB} 1^{\star} 03$. It was not possible to ascertain the ethnic origin of the patients with sclerosing lymphocytic lobulitis, whereas the control population was white. HLA-DRB $1^{\star} 04$ is seen at higher frequencies in white populations, compared with other ethnic groups. ${ }^{17} \mathrm{~A}$ different ethnic composition of the patient and control groups is therefore unlikely to lead to the results observed. On the contrary, it would tend to cause a reduction of the difference in frequency of HLA-DRB $1{ }^{\star} 04$ between patients and controls. Sclerosing lymphocytic lobulitis is associated with insulin dependent diabetes mellitus, so we therefore also looked at the frequencies of two alleles associated with diabetes, finding an increased frequency of HLA-DQB $1{ }^{\star} 0302$, but not of $\mathrm{DQB} 1^{\star} 02$.

The two HLA class II genotypes that were present at increased frequencies-DRB1 ${ }^{\star} 04$ and $\mathrm{DQB} 1^{\star} 0302$ - are in linkage disequilibrium and are both associated with insulin dependent diabetes mellitus. Comparison of the frequencies of these genotypes in patients with and without diabetes suggested that the increased frequencies of HLA- DRB $1^{\star} 04$ and DQB $1^{\star} 0302$ in the patient group as a whole reflected the strong link with diabetes. There was a non-significant increase in the frequency of DRB $1^{\star} 04$ in patients without diabetes compared with controls $(50 \%$ v 33\%). Interestingly, DQB ${ }^{\star} 0301$ (also found in linkage disequilibrium with $\mathrm{DRB} 1{ }^{\star} 04^{17}$ but not associated with insulin dependent diabetes mellitus $^{23}$ ) showed no increased frequency in the sclerosing lymphocytic lobulitis patients with or without diabetes. Larger series are required to investigate further whether HLA$\mathrm{DRB} 1{ }^{\star} 04$ is present at increased frequencies in non-diabetic patients with sclerosing lymphocytic lobulitis. However, large series of patients with sclerosing lymphocytic lobulitis are difficult to collect as the condition is uncommon and may be under-recognised. The present study includes more non-diabetic patients (19) than any previous series, the largest of which were those of Lammie et al (10 non-diabetic patients; some included in the present study) and Schwartz and Strauchen (seven non-diabetic patients). ${ }^{12}$

In conclusion, these results provide further evidence of a link between sclerosing lymphocytic lobulitis and insulin dependent diabetes mellitus, supported by HLA class II genotypic associations, consistent with an autoimmune aetiology. Further study is needed to investigate whether the frequency of HLA$\mathrm{DRB} 1{ }^{\star} 04$ is increased in patients with sclerosing lymphocytic lobulitis but without diabetes.

The methods used in this study were developed with the support of the Wessex Cancer Trust.

1 Lammie GA, Bobrow LG, Staunton MDM, et al. Sclerosing ymphocytic lobulitis of the breast-evidence for an autoimmune pathogenesis. Histopathology 1991;19:13-20.

2 Schwartz IS, Strauchen JA. Lymphocytic mastopathy. An autoimmune disease of the breast? Am f Clin Pathol 1990; 93:725-30.

3 Hyjek E, Isaacson PG. Primary B cell lymphoma of the thyroid and its relationship to Hashimoto's thyroiditis. Hum Pathol 1988;19:1315-26.

4 Andrade RE, Hagen KA, Manivel JC. Distribution and immunophenotype of the inflammatory cell population in the benign lymphoepithelial lesion (Mikulicz's disease). Hum Pathol 1988;19:932-41.

5 Atkinson MA, MacLaren NK. The pathogenesis of insulindependent diabetes mellitus. $N$ Engl f Med 1994;331: 1428-36.

6 Soler NG, Khardori R. Fibrous disease of the breast, hyroiditis, and cheiroarthropathy in type I diabetes mellitus. Lancet 1984;i:193-5.

7 Byrd BF, Hartmann WH, Graham LS, et al. Mastopathy in insulin-dependent diabetics. Ann Surg 1987;205:529-32.

8 Thorsby E. HLA associated diseases. Hum Immunol 1997;53:1-11.

9 Brodsky FM, Lem L, Bresnahan PA. Antigen processing and presentation. Tissue Antigens 1996;47:464-71.

10 Marsh SGE. HLA class II region sequences, 1998. Tissue Antigens 1998;51:467-507.

11 Stern LJ, Wiley DC. Antigenic peptide binding by class I and class II histocompatibility proteins. Structure 1994;2: $245-51$

12 Cardaba B, de Pablo R, Vilches C, et al. Allergy to olive pollen: T-cell response from olive allergic patients is restricted by DR7-DQ2 antigens. Clin Exp Allergy 1996;26; $316-22$

13 Lundin KEA, Gjertsen HA, Scott H, et al. Function of DQ2 and DQ8 as HLA susceptibility molecules in celiac disease. Hum Immunol 1994;41:24-7.

14 Bunce M, Young NT, Welsh KI. Molecular HLA typingthe brave new world. Transplantation 1997;64:1505-13.

15 Bateman AC, Leung ST, Howell WM, et al. Detection of specimen contamination in routine histopathology by HLA class II typing using the polymerase chain reaction and sequence specific oligonucleotide probing. F Pathol 1994; 173:243-8.

16 Howell WM, Leung ST, Jones DB, et al. HLA-DRB, -DQA and -DQB polymorphism in celiac disease and enteropathy-associated T-cell lymphoma. Common features and additional risk factors for malignancy. Hum Immunol 1995;43:29-37.

17 Imanishi T, Akaza T, Kimura A, et al. Allele and haplotype frequencies for HLA and complement loci in various ethnic
groups. In: Tsuji K, Aizawa M, Sasazuki T, eds. HLA 1991 . Proceedings of the Eleventh International Histocompatibility Workshop and Conference, vol 1. Oxford: Oxford University Press, 1992:1065-220.

18 Williams PH, Rubin CME, Theaker JM. Sclerosing lymphocytic lobulitis of the breast. Clin Radiol 1995;50: lymphocy.

19 Kimura A, Sasazuki T. Eleventh International Histocompatibility Workshop reference protocol for the HLA DNAtyping technique. In: Tsuji K, Aizawa M, Sasazuki T, eds. HLA 1991. Proceedings of the Eleventh International Histocompatibility Workshop and Conference, vol 1. Oxford: Oxford University Press, 1992:397-439.

20 Nevinny-Stickel C, Hinzpeter M, Andreas A, et al. Non-radioactive oligotyping for HLA-DR1-DRw10 using polymerase chain reaction, digoxigenin-labelled oligonucleotides and chemoluminescence detection. Eur f Immunogenet 1991;18:323-32.

21 Bateman AC, Hemmatpour SK, Theaker JM, et al. Nested polymerase chain reaction-based HLA class II typing for
the unique identification of formalin-fixed and paraffinthe unique identification of formalin-fixed 
22 Dyer P, Warrens A. Design and interpretation of studies of the major histocompatibility complex in disease. In: Lechler R, ed. HLA and disease. London: Academic Press, 1994 93-121.

23 Ronningen, KS, Spurkland A, Tait BD, et al. HLA class II associations in insulin-dependent diabetes among Blacks, Caucasoids and Japanese. In: Tsuji K, Aizawa M, Sasazuki $\mathrm{T}$, eds. HLA 1991. Proceedings of the Eleventh International
Histocompatibility Workshop and Conference, vol 1. Oxford: Oxford University Press, 1992:713-22.

4 Tomaszewski JE, Brooks JSJ, Hicks D, et al. Diabetic mastopathy: a distinctive clinicopathologic entity. Hum Pathol 1992;23:780-6.

25 Ashton MA, Lefkowitz M, Tavassoli FA. Epithelioid stromal cells in lymphocytic mastitis - a source of confusion with invasive carcinoma. Mod Pathol 1994;7:49-54.

\section{Fournal of Clinical Pathology - http://www.jclinpath.com}

Visitors to the world wide web can now access the fournal of Clinical Pathology either through the BMJ Publishing Group's home page (http://www.bmjpg.com) or directly by using its individual URL (http://www.jclinpath.com). There they will find the following:

- Current contents list for the journal

- Contents lists of previous issues

- Members of the editorial board

- Information for subscribers

- Instructions for authors

- Details of reprint services.

A hotlink gives access to:

- BMJ Publishing Group home page

- British Medical Association web site

- Online books catalogue

- BMJ Publishing Group books.

The web site is at a preliminary stage and there are plans to develop it into a more sophisticated site. Suggestions from visitors about features they would like to see are welcomed. They can be left via the opening page of the BMJ Publishing Group site or, alternatively, via the journal page, through "about this site". 